International Journal of Humanoid Robotics

Vol. 10, No. 4 (2013) 1399001 (4 pages)

(C) World Scientific Publishing Company

DOI: $10.1142 /$ S0219843613990016

\title{
Author Index \\ Volume 10 (2013)
}

Abels, A. and Kruusmaa, M., Shape Control of an Anthropomorphic Tailoring Robot Mannequin

Adiwahono, A. H., Chew, C.-M. and Liu, B., Push Recovery Through Walking Phase Modification for Bipedal Locomotion

Ahn, H. S., Lee, D.-W., Choi, D., Lee, D.-Y., Lee, H.-G. and Baeg,

M.-H., Development of an Incarnate Announcing Robot System

Using Emotional Interaction with Humans

Al Moubayed, S., see Mirnig, N.

Aleotti, J. and Caselli, S., Functional Principal Component Analysis for Recognition of Arm Gestures and Humanoid Imitation

Alina, R., see Anamaria, P. C.

Anamaria, P. C., Ramona, S., Sebastian, P., Jelle, S., Alina, R.,

Daniel, D., Johan, V., Dirk, L. and Bram, V., Can the Social Robot

Probo Help Children with Autism to Identify Situation-Based

Emotions? A Series of Single Case Experiments

Aoustin, Y. and Formalskii, A., Upward Jump of a Biped

Arai, T., see Ratsamee, P.

Baeg, M.-H., see Ahn, H. S.

Bennewitz, M., see Maier, D.

Beskow, J., see Mirnig, N.

Beskow, J., see Moubayed, S. A.

Bin Hammam, G., see Wensing, P. M.

Borovac, B., see Nikolić, M.

Borst, C., see Zacharias, F.

Bram, V., see Anamaria, P. C.

Bugmann, G., see Ford, C. C.

Caselli, S., see Aleotti, J.

Castellano, G., Leite, I., Pereira, A., Martinho, C., Paiva, A. and

McOwan, P. W., Multimodal Affect Modeling and Recognition for

Empathic Robot Companions

Chen, Q., see Zhang, W.

Chetcuti, A., see Saliba, M. A.

Chew, C.-M., see Adiwahono, A. H.

Chi, S.-W., see Kuo, C.-H.

Chinellato, E., see Stenzel, A.

Cho, B.-K., Kim, J.-H. and Oh, J.-H., Balancing Strategy Using the

Principle of Energy Conservation for a Hopping Humanoid Robot

Choi, D., see Ahn, H. S.

Chou, H.-C., see Kuo, C.-H.

Culverhouse, P., see Ford, C. C.

$10(2013) 1350002$

$10(2013) 1350022$

10 (2013) 1350017

10 (2013) 1350011

$10(2013) 1350033$

10 (2013) 1350025

10 (2013) 1350025

10 (2013) 1350032

10 (2013) 1350008

10 (2013) 1350017

10 (2013) 1350016

10 (2013) 1350011

10 (2013) 1350005

10 (2013) 1350027

10 (2013) 1350012

10 (2013) 1350031

10 (2013) 1350025

10 (2013) 1350006

10 (2013) 1350033

10 (2013) 1350010

10 (2013) 1330001

10 (2013) 1350001

10 (2013) 1350022

10 (2013) 1350021

10 (2013) 1350015

10 (2013) 1350020

10 (2013) 1350017

10 (2013) 1350021

10 (2013) 1350006 
Daniel, D., see Anamaria, P. C.

10 (2013) 1350025

Dariush, B., see Wensing, P. M.

10 (2013) 1350027

Del Pobil, A. P., see Stenzel, A.

10 (2013) 1350015

Dirk, L., see Anamaria, P. C.

10 (2013) 1350025

$\mathrm{Du}, \mathrm{D}$., see Zhang, W.

Endo, N., see Trovato, G.

Fan, S., see Zhang, T.

Farrugia, M. J., see Saliba, M. A.

Ford, C. C., Bugmann, G. and Culverhouse, P., Modeling the Human

10 (2013) 1330001

10 (2013) 1350013

10 (2013) 1350028

10 (2013) 1350001 Blink: A Computational Model for Use Within Human-Robot

\section{Interaction}

Formalskii, A., see Aoustin, Y.

Fujinami, T., see Yamazaki, R.

Gams, A., see Petrič, T.

Granström, B., see Mirnig, N.

Gustafson, J., see Mirnig, N.

Hagita, N., see Liu, C.

Hashimoto, K., see Trovato, G.

Hashimoto, M., see Li, Y.

Havangi, R., Teshnehlab, M., Nekoui, M. A. and Taghirad, H. D., A Novel Particle Filter Based SLAM

Hirzinger, G., see Zacharias, F.

Ishi, C. T., see Liu, C.

Ishiguro, H., see Yamazaki, R.

Ishiguro, H., see Liu, C.

Jelle, S., see Anamaria, P. C.

Jiang, L., see Zhang, T.

Johan, V., see Anamaria, P. C.

Karssen, J. G. D., see van Oijen, T. P.

Kim, J.-H., see Cho, B.-K.

Kim, J.-Y., see Lee, S.

Kim, J.-Y. and Kim, Y.-S., Whole-Body Motion Generation of Android Robot Using Motion Capture and Nonlinear Constrained Optimization

Kim, M., see Lee, S.

Kim, Y.-S., see Kim, J.-Y.

Kishi, T., see Trovato, G.

Kruusmaa, M., see Abels, A.

Kuo, C.-H., Chou, H.-C., Chi, S.-W. and Lien, Y.-D., Vision-Based Obstacle Avoidance Navigation with Autonomous Humanoid

Robots for Structured Competition Problems

Lappe, M., see Stenzel, A.

Lee, D.-W., see Ahn, H. S.

Lee, D.-Y., see Ahn, H. S.

Lee, H.-G., see Ahn, H. S.

Lee, S., Kim, J.-Y. and Kim, M., Development and Walking Control of Emotional Humanoid Robot, KIBO

Leite, I., see Castellano, G.

Li, Y. and Hashimoto, M., Emotional Synchronization-Based

Human-Robot Communication and Its Effects

10 (2013) 1350006

10 (2013) 1350032

10 (2013) 1350007

10 (2013) 1350029

10 (2013) 1350011

10 (2013) 1350011

10 (2013) 1350009

10 (2013) 1350013

10 (2013) 1350014

10 (2013) 1350018

10 (2013) 1350031

10 (2013) 1350009

10 (2013) 1350007

10 (2013) 1350009

10 (2013) 1350025

10 (2013) 1350028

10 (2013) 1350025

10 (2013) 1350004

10 (2013) 1350020

10 (2013) 1350024

10 (2013) 1350003

10 (2013) 1350024

10 (2013) 1350003

10 (2013) 1350013

10 (2013) 1350002

10 (2013) 1350021

10 (2013) 1350015

10 (2013) 1350017

10 (2013) 1350017

10 (2013) 1350017

10 (2013) 1350024

10 (2013) 1350010

10 (2013) 1350014 
Lien, Y.-D., see Kuo, C.-H.

10 (2013) 1350021

Liepelt, R., see Stenzel, A.

10 (2013) 1350015

Liu, B., see Adiwahono, A. H.

Liu, C., Ishi, C. T., Ishiguro, H. and Hagita, N., Generation of

10 (2013) 1350022

Nodding, Head Tilting and Gazing for Human-Robot Speech Interaction

Liu, H., see Zhang, T.

Liu, X., see Zhan, Q.

Mae, Y., see Ratsamee, P.

Maier, D., Stachniss, C. and Bennewitz, M., Vision-Based Humanoid Navigation Using Self-Supervised Obstacle Detection

Martinho, C., see Castellano, G.

Matsumura, K., see Yamazaki, R.

McOwan, P. W., see Castellano, G.

Minato, T., see Yamazaki, R.

Mirnig, N., Weiss, A., Skantze, G., Al Moubayed, S., Gustafson, J., Beskow, J., Granström, B. and Tscheligi, M., Face-to-Face with a Robot: What Do We Actually Talk About?

Mohareri, O. and Rad, A. B., A Vision-Based Location Positioning System via Augmented Reality: An Application in Humanoid Robot Navigation

Moubayed, S. A., Skantze, G. and Beskow, J., The Furhat BackProjected Humanoid Head-Lip Reading, Gaze and Multi-Party Interaction

Nekoui, M. A., see Havangi, R.

Nemec, B., see Petrič, T.

Nikolić, M., Borovac, B., Raković, M. and Savić, S., A Further Generalization of Task-Oriented Control Through Tasks Prioritization

Nishikawa, M., see Yamazaki, R.

Nishio, S., see Yamazaki, R.

Ogawa, K., see Yamazaki, R.

Oh, J.-H., see Cho, B.-K.

Ohara, K., see Ratsamee, P.

Orin, D. E., see Wensing, P. M.

Paiva, A., see Castellano, G.

Pereira, A., see Castellano, G.

Peternel, L., see Petrič, T.

Petrič, T., Peternel, L., Gams, A., Nemec, B. and Žlajpah, L., Navigation Methods for the Skiing Robot

Rad, A. B., see Mohareri, O.

Raković, M., see Nikolić, M.

Ramona, S., see Anamaria, P. C.

Ratsamee, P., Mae, Y., Ohara, K., Takubo, T. and Arai, T., Human-Robot Collision Avoidance Using a Modified Social Force Model with Body Pose and Face Orientation

10 (2013) 1350009

10 (2013) 1350028

10 (2013) 1350026

$10(2013) 1350008$

10 (2013) 1350016

10 (2013) 1350010

$10(2013) 1350007$

10 (2013) 1350010

10 (2013) 1350007

10 (2013) 1350011

10 (2013) 1350019

10 (2013) 1350005

10 (2013) 1350018

10 (2013) 1350029

10 (2013) 1350012

10 (2013) 1350007

10 (2013) 1350007

10 (2013) 1350007

10 (2013) 1350020

10 (2013) 1350008

10 (2013) 1350027

10 (2013) 1350010

10 (2013) 1350010

10 (2013) 1350029

10 (2013) 1350029

10 (2013) 1350019

10 (2013) 1350012

10 (2013) 1350025

Saliba, M. A., Chetcuti, A. and Farrugia, M. J., Towards the Rationalization of Anthropomorphic Robot Hand Design: Extracting Knowledge from Constrained Human Manual Dexterity Testing

$10(2013) 1350008$

$10(2013) 1350001$ 
Savić, S., see Nikolić, M.

Sebastian, P., see Anamaria, P. C.

10 (2013) 1350012

Skantze, G., see Moubayed, S. A.

10 (2013) 1350025

Skantze, G., see Mirnig, N.

$10(2013) 1350005$

10 (2013) 1350011

Stachniss, C., see Maier, D.

Stenzel, A., Chinellato, E., Del Pobil, A. P., Lappe, M.

10 (2013) 1350016

and Liepelt, R., How Deeply Do We Include Robotic Agents in the Self?

Sun, Z., see Zhang, W.

Taghirad, H. D., see Havangi, R.

Takanishi, A., see Trovato, G.

Takubo, T., see Ratsamee, P.

Teshnehlab, M., see Havangi, R.

Tondu, B., A Zonotope-Based Approach for Manipulability Study of Redundant Robot Limbs

Tondu, B., Closed-Form Working Volume Computation and Structural Optimization of Redundant Serial Robot Chains Derived from Zonotope Theory

10 (2013) 1350015

10 (2013) 1330001

10 (2013) 1350018

10 (2013) 1350013

10 (2013) 1350008

10 (2013) 1350018

$10(2013) 1350023$

$10(2013) 1350030$

Trovato, G., Zecca, M., Kishi, T., Endo, N., Hashimoto, K. and Takanishi, A., Generation of Humanoid Robot's Facial

Expressions for Context-Aware Communication

Tscheligi, M., see Mirnig, N.

van Oijen, T. P., Karssen, J. G. D. and Wisse, M., The Effect of Center of Mass Offset on the Disturbance Rejection of Running Robots

Weiss, A., see Mirnig, N.

Wensing, P. M., Bin Hammam, G., Dariush, B. and Orin, D. E., Optimizing Foot Centers of Pressure Through Force Distribution in a Humanoid Robot

Wisse, M., see van Oijen, T. P.

Wolf, S., see Zacharias, F.

Yamazaki, R., Nishio, S., Ogawa, K., Matsumura, K., Minato, T., Ishiguro, H., Fujinami, T. and Nishikawa, M., Promoting Socialization of Schoolchildren Using a Teleoperated Android: An Interaction Study

Zacharias, F., Borst, C., Wolf, S. and Hirzinger, G., The Capability Map: A Tool to Analyze Robot Arm Workspaces

Zecca, M., see Trovato, G.

Zhang, T., Fan, S., Jiang, L. and Liu, H., Development and Experiment Analysis of Anthropomorphic Prosthetic Hand with Flexible Three-Axis Tactile Sensor

10 (2013) 1350013

10 (2013) 1350011

10 (2013) 1350004

10 (2013) 1350011

10 (2013) 1350027

10 (2013) 1350004

10 (2013) 1350031

10 (2013) 1350007

10 (2013) 1350031

10 (2013) 1350013

$10(2013) 1350028$

Zhang, W., Zhao, D., Zhou, H., Sun, Z., Du, D. and Chen, Q., TwoDOF Coupled and Self-Adaptive (COSA) Finger: A Novel UnderActuated Mechanism

Zhan, Q. and Liu, X., Hand Grasp Function Analysis Based on VF Set

Zhao, D., see Zhang, W.

Zhou, H., see Zhang, W.

Žlajpah, L., see Petrič, T.

10 (2013) 1330001

10 (2013) 1350026

10 (2013) 1330001

10 (2013) 1330001

10 (2013) 1350029 